nuclear-envelope proteins, cause autosomal recessive axonal neuropathy in human (Charcot-MarieTooth disorder type 2) and mouse. Am J Hum Genet 2002; 70: 726-36.

6. Bonne G, Di Barletta MR, Varnous $S$, et al. Mutations in the gene encoding lamin $A / C$ cause autosomal dominant Emery-Dreifuss muscular dystrophy. Nat Genet 1999; $21: 285-8$.
7. Vigouroux C, Auclair M, Dubosclard $\varepsilon$, et al. Nuclear envelope disorganization in fibroblasts from lipodystrophic patients with heterozygous $\mathrm{R} 482 \mathrm{p} / \mathrm{W}$ mutations in the lamin $\mathrm{A} / \mathrm{C}$ gene. J Cell Sci 2001; 114 : 4459-68.

8. Östlund C, Bonne G, Schwartz K, Worman HJ. Properties of lamin A mutants found in Emery-
Dreifuss muscular dystrophy, cardiomyopathy and Dunnigan-type partial lipodystrophy. J Cell Sci 2001 ; 114: 14435-45.

9. Krimm I, Ostlund C, Gilquin $B$, et al. The Ig-like structure of the $\mathrm{C}$-terminal domain of lamin a/c, mutated in muscular dystrophies cardiomyopathy, and partial lipodystrophy. Structure (Camb) 2002; 10: 811-23
10. Stuurman N, Heins S, Aebi U. Nuclear lamins: their structure, assembly, and interactions.

J Struct Biol 1998; 122: 42-66.

11. Cohen M, Lee KK, Wilson KL, Gruenbaum Y.

Transcriptional repression, apoptosis, human disease and the functional evolution of the nuclear lamina. Trends Biochem Sci 2001; 26: 41-7.

\title{
NOUVELLE
}

\section{Vers la rythmologie moléculaire et informatique}

\author{
Denis Escande
}

$>$ La cause la plus fréquente de décès dans la population adulte des pays industrialisés demeure la mort subite liée à la maladie coronarienne. Son incidence est de l'ordre de 0,36-1,28 cas annuels pour 1000 habitants [1]. Dans 75-80\% des cas de mort subite d'origine cardiovasculaire, le rythme cardiaque enregistré est la fibrillation ventriculaire, la forme la plus grave des troubles du rythme. Parmi les troubles du rythme chroniques ne mettant pas en jeu le pronostic vital immédiat, la fibrillation auriculaire est de loin la plus fréquente avec une prévalence de $1 / 1000$ dans la population adulte, en augmentation constante du fait du vieillissement de la population dans les pays développés.

La rythmologie a pour champs d'action les anomalies du rythme cardiaque: la rythmologie clinique assure leur diagnostic et leur traitement; la rythmologie expérimentale a pour ambition de comprendre leur genèse et d'identifier de nouvelles voies thérapeutiques. La rythmologie clinique s'appuie sur l'électrocardiographie de surface et endocavitaire. La rythmologie expérimentale dispose d'un continuum de techniques électrophysiologiques depuis l'étage moléculaire (patch-clamp), cellulaire (patch-clamp et enregistrement des potentiels d'action) jusqu'à l'étage intégré (électrocardiographie). L'ECG (électrocardiogramme) représente l'intégration spatio-temporelle la plus achevée et la plus complexe du fonctionnement répétitif de protéinescanal dont l'activité unitaire est mesurable et dont la nature moléculaire est de mieux en mieux connue.

\section{Rythmologie et génome}

Comme les autres disciplines, la rythmologie a largement bénéficié de la révolution du génome. Les gènes codant pour la plupart des canaux ioniques ont été identifiés par des approches de génétique inverse, ou par analogie de structure de leurs produits. Ces découvertes ont démontré la complexité du transcriptome ionique. Pas moins de soixante-quinze gènes différents codant pour des canaux potassiques ont été recensés dans le génome humain. En parallèle avec le décryptage du génome et l'identification de la localisation chromosomique de nombreux gènes, des maladies génétiques responsables de troubles du rythme ou de la conduction (canalopathies) ont été individualisées et leur gène morbide identifié [2]. Il en est ainsi: (1) du syndrome du QT long [3] dont on connaît au moins 5 formes génétiques (LQT1-5) et qui est lié à des mutations de gènes codant pour des canaux potassiques (LQT1 et LQT2), le canal sodique cardiaque (LQT3), ou des régulateurs de canaux (LQT4 et LQT5); (2) du syndrome de Brugada [4] responsable de fibrillations ventriculaires idiopathiques et lié à des mutations du gène SCN5A codant pour le canal sodique cardiaque; (3) des tachycardies ventriculaires catécholaminergiques [3] liées à des mutations du gène codant pour le canal calcique du réticulum sarcoplasmique; (4) de la forme génétique de la maladie de Lenègre [5] liée à une mutation de SCN5A. On peut remarquer que des mutations dans un même gène ( $S C N 5 A$ ) peuvent donner lieu à des phénotypes rythmologiques très différents. La perte de fonction de SCN5A conduit à des troubles de la conduction de l'influx (ce qui n'est pas étonnant étant donné le rôle central du courant sodique dans la conduction), mais aussi à de graves troubles du rythme ventriculaire. On peut rapprocher de cette dernière situation les effets pro-arythmiques paradoxaux des médicaments anti-arythmiques de classe I dont le mode d'action commun est une inhibition du courant sodique. Par ailleurs, il est remarquable de noter que les différents gènes impliqués dans le syndrome du QT long congénital ne produisent pas exactement les mêmes anomalies électrocardiographiques et qu'un 
œil exercé est capable dans $70 \%$ des cas de faire le diagnostic du gène muté sur l'aspect de la repolarisation. Rappelons que l'espace QT de l'ECG est le reflet, au niveau du cœur entier, de la repolarisation des cellules du myocarde ventriculaire (voir encadré). Au niveau cellulaire, le potentiel d'action est la résultante d'une cascade de transferts de charges ioniques qui se font soit à travers des structures protéiques, les canaux ioniques, soit par l'intermédiaire de systèmes d'échanges. Les transferts de charges peuvent se faire dans le sens d'une entrée d'ions positifs créant un courant dépolarisant (courants sodique ou calcique) ou à l'inverse d'une sortie d'ions positifs créant un courant repolarisant (courants potassique ou chlore). Ainsi, la repolarisation cellulaire est retardée et l'espace QT allongé, si le courant net repolarisant est diminué.

\section{La rythmologie de la souris}

Comme dans les autres disciplines, le modèle murin s'est progressivement imposé en rythmologie du fait des possibilités offertes par la manipulation génétique [6]. II est spectaculaire de constater que l'ensemble des moyens d'investigation électrophysiologique (incluant l'électrophysiologie invasive intracavitaire et la télémétrie) ont été adaptés à un organisme dont le poids du cœur n'excède pas 100 mg (contre $450 \mathrm{~g}$ chez l'homme). Cependant, le cœur de souris n'est certainement pas le meilleur modèle électrophysiologique: sa fréquence cardiaque de base est de 600 $\mathrm{cpm}$ et les courants ioniques impliqués dans la repolarisation sont très différents de ceux du cœur humain. En dépit de ces différences et surtout en dépit de sa très petite taille, le modèle murin a démontré sa capacité à produire des anomalies rythmiques proches de celles qui sont observées chez l'homme (Figure 1). II offre l'opportunité d'étudier les effets de la manipulation génique sur les troubles du rythme et de la conduction.

\section{Une application pratique}

de la rythmologie moléculaire:

le dépistage des effets

pro-arythmiques des substances

\section{pharmacologiques}

Plus de la moitié des médicaments qui ont été retirés du marché depuis 1998 l'ont été parce que ces médicaments créent des troubles du rythme cardiaque [7]. Cet effet, qui concerne de nombreuses classes de médicaments (voir à ce sujet http://www.qtdrugs.org/), est lié dans la majorité des cas à un allongement de la repolarisation cardiaque (espace DT de l'électrocardiogramme, voir encadré). Les accidents rythmologiques induits par les médicaments ont une incidence si faible (mais une gravité extrême) qu'ils ne sont généralement pas dépistés au cours des essais cliniques nécessaires à la constitution du dossier d'enregistrement. L'électrophysiologie moléculaire a apporté une solution pratique à ce problème. En effet, il a été démontré que les substances qui allongent de façon incidente la repolarisation cardiaque sont des bloqueurs des canaux $\mathrm{K}^{+} \mathrm{H} \varepsilon R \mathrm{RG}$ (human ether a go-go related gene). préalablement identifiés par une approche de génétique inverse comme étant responsables du syndrome du PT long congénital LQT2. Ainsi, la « recommandation » des agences américaine et européenne est dorénavant que l'affinité pour le canal HERG de toute nouvelle entité chimique soit évaluée par les techniques d'électrophysiologie moléculaire avant toute administration à l'homme.

Le stade ultime:

l'intégration informatique/

le physiome

L'électrophysiologie, discipline à la frontière entre la physiologie et la biophysique, propose une approche quantitative $\mathrm{du}$ fonctionnement cellulaire. Chaque courant ionique peut être caractérisé par un ensemble d'équations plus ou moins complexes qui décrivent ses propriétés élémentaires (activation, inactivation, réactivation...). L'ensemble des équations rapportant les caractéristiques de l'ensemble des courants d'une cellule cardiaque permet de reconstituer par informatique son activité électrique élémentaire: le potentiel d'action. L'intégration des différents signaux électriques issus d'un syncytium tridimensionnel de cellules de caractéristiques électrophysiologiques variées (les courants ioniques ont une répartition inhomogène dans l'épaisseur du myocarde; [8]) permet de reconstituer par informatique un tracé électrocardiographique de surface [9]. Ces approches informatiques sont d'une utilité certaine à la compréhension des mécanismes cellulaires et surtout multicellulaires qui engendrent les troubles du rythme. A un stade ultime, on peut imaginer ajouter à cette intégration électrophysiologique les données multiples issues des analyses du transcriptome et du protéome qui décrivent les niveaux d'expression respectifs des canaux ioniques, de leurs variants d'épissage et de leurs nombreux régulateurs dans une situation physiologique et dans une situation pathologique (remodelage). L'aboutissement de cette démarche (physiome) permettra l'intégration quantitative des données du génome et de la physiologie [10]. Elle sera certainement d'une importance majeure dans le processus de développement du médicament. $\checkmark$

Towards molecular and computational rhythmology
Figure 1. Enregistrement télémétrique d'une torsades-de-pointe induite par l'érythromycine chez une souris transgénique présentant un syndrome du PT long. Nathalie LeBerre, Alain Corbier, Sophie Demolombe (Aventis et Inserm U.533).

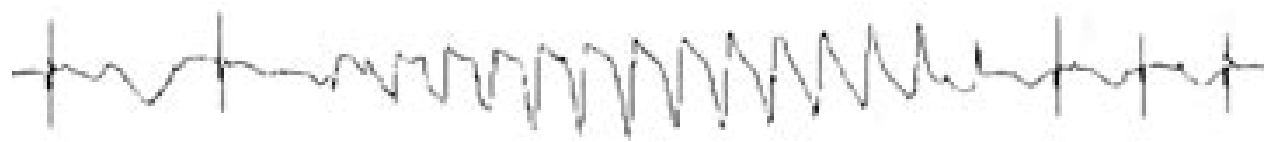




\section{SYNDROME DU PT LONG}

Le phénotype du syndrome du QT long congénital est caractérisé par un espace QT anormalement long à l'ECG. La découverte de la maladie concerne souvent des sujets jeunes. II existe une prédominance féminine, qui a été retrouvée dans le registre international et dans plusieurs études cliniques où le critère de diagnostic était purement électrocardiographique. Cette prédominance féminine pourrait s'expliquer par le fait que les femmes ont physiologiquement un espace PT plus long que celui des hommes. Les sujets atteints ont une bradycardie sinusale relative. Le problème majeur posé par ce syndrome est que certains sujets porteurs de l'anomalie génétique feront des syncopes par trouble du rythme ventriculaire pouvant conduire à la mort subite. II s'agit de tachycardies ventriculaires polymorphes paroxystiques, entrecoupées de retour en rythme sinusal, ayant toutes les caractéristiques des torsades de pointes. Les syncopes apparaissent habituellement dans des situations de stress, surtout lors d'une émotion mais peuvent aussi apparaître au repos (source: site http://www.ifr26.nantes. inserm.fr).

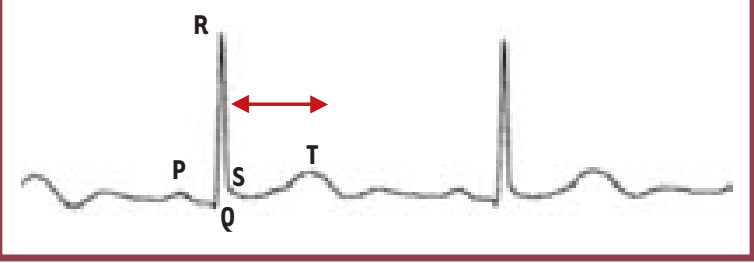

\section{RÉFÉRENCES}

1. Vreede-Swagemakers JJ, Gorgels AP, Dubois-Arbouw WI, et al. Out-of-hospital cardiac arrest in the 1990's: a population-based study in the Maastricht area on incidence, characteristics and survival. J Am Coll Cardiol 1997; 30: 1500-5.

2. Marban E. Cardiac channelopathies. Nature 2002; 415: 213-8

3. Towbin JA, Vatta M, Li $H$. Genetics of Brugada, long QT, and arrhythmogenic right ventricular dysplasia syndromes. J Electrocardiol 2000; 33 Suppl: 11-22.

4. Naccarelli GV, Antzelevitch C. The Brugada syndrome: clinical, genetic, cellular, and molecular abnormalities. Am J Med 2001; 110: 573-81.

5. Schott JJ, Alshinawi C, Kyndt $F$, et al. Cardiac conduction defects associate with mutations in SCN5A. Nat Genet 1999; $23: 20-1$.

6. Nerbonne JM, Nichols CG, Schwarz TL, Escande D. Genetic manipulation of cardiac $\mathrm{K}^{+}$channel function in mice: what have we learned, and where do we go from here? Circ Res 2001; 89: 944-56.

7. Haverkamp W, Breithardt G, Camm AJ, et al. The potential for $\mathrm{QT}$ prolongation and proarrhythmia by non-antiarrhythmic drugs: clinical and regulatory implications. Report on a Policy Conference of the European Society of Cardiology. Cardiovasc Res 2000; 47 : 219-33.

8. Schram G, Pourrier M, Melnyk P, Nattel S. Differential distribution of cardiac ion channel expression as a basis for regional specialization in electrical function. Circ Res 2002; 90 : 939-50.

9. Gima K, Rudy Y. Ionic current basis of electrocardiographic waveforms: a model study. Circ Res 2002; 90 : 889-96.

10. Noble D. Modeling the heartfrom genes to cells to the whole organ. Science 2002; 295: 1678-82.

\section{NOUVELLE}

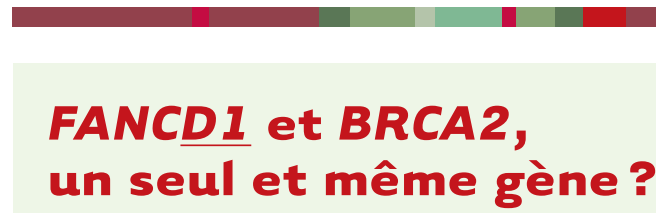

Dora Papadopoulo, Ethel Moustacchi
Cnrs UMR 218, Institut Curie, Section de Recherche, 26, rue d'Ulm, 75248 Paris Cedex 05, France.

présence du complexe est nécessaire à l'activation de la protéine FancD2 en une isoforme mono-ubiquitinylée FancD2-L qui, dans les cellules normales, s'accumule dans des foyers nucléaires en réponse à des dommages spécifiques de l'ADN [4]. De plus, il a été démontré que FancD2 est phosphorylée par le produit du gène ATM (ataxia telangiectasia mutated) et cette phosphorylation est nécessaire pour le contrôle de la progression de la phase $S$ du cycle cellulaire en présence de dommages induits par les radiations ionisantes (Figure 1) [5].

Le premier rapprochement entre des gènes 